\title{
A branch-and-price algorithm for the long-term home care scheduling problem
}

\author{
Gamst, Mette; Jensen, Thomas Sejr
}

Published in:

Operations Research Proceedings 2011

Link to article, DOI:

10.1007/978-3-642-29210-1_77

Publication date:

2012

Link back to DTU Orbit

Citation (APA):

Gamst, M., \& Jensen, T. S. (2012). A branch-and-price algorithm for the long-term home care scheduling problem. In D. Klatte, H-J. Lüthi, \& K. Schmedders (Eds.), Operations Research Proceedings 2011: Selected Papers of the International Conference on Operations Research (pp. 483-488). Springer. Operations Research Proceedings https://doi.org/10.1007/978-3-642-29210-1_77

\section{General rights}

Copyright and moral rights for the publications made accessible in the public portal are retained by the authors and/or other copyright owners and it is a condition of accessing publications that users recognise and abide by the legal requirements associated with these rights.

- Users may download and print one copy of any publication from the public portal for the purpose of private study or research.

- You may not further distribute the material or use it for any profit-making activity or commercial gain

- You may freely distribute the URL identifying the publication in the public portal

If you believe that this document breaches copyright please contact us providing details, and we will remove access to the work immediately and investigate your claim. 


\title{
A branch-and-price algorithm for the long-term home care scheduling problem
}

\author{
M. Gamst and T. Sejr Jensen
}

\begin{abstract}
In several countries, home care is provided for certain citizens living at home. The long-term home care scheduling problem is to generate work plans such that a high quality of service is maintained, the work hours of the employees are respected, and the overall cost is kept as low as possible. We propose a branchand-price algorithm for the long-term home care scheduling problem. The pricing problem generates a one-day plan for an employee, and the master problem merges the plans with respect to regularity constraints. The method is capable of generating plans with up to 44 visits during one week.
\end{abstract}

\section{Introduction}

In several countries, home care is provided for certain citizens living at home. Home care offers cleaning, grocery shopping, help with personal hygiene and medicine, etc. The long-term home care scheduling problem is to generate work plans spanning a longer period of time, such that a high quality of service is maintained, the work hours of the employees are respected, and the overall cost is kept as low as possible.

Quality of service consists of the following. Regularity: all visits at a citizen should be conducted at the same time of the day and by the same (small group of) employee(s) in order for the citizen to feel safe. Skill set requirements: certain tasks can only be performed by a subset of the employees due to skill requirements.

All time windows are soft, i.e., preferred visit times and employee work hours can be violated at a cost. Such violations are denoted "busyness". The overall cost of a solution consists of a linear combination of travel time between visits, quality of service, and busyness.

The long-term home care scheduling problem is $N P$-hard and is typically approached in one of two ways in the literature: (1) plans for employees for a single day are generated. This corresponds to a modified VRPTW and is denoted the daily

University of Southern Denmark, DK-5230 Odense M, e-mail: gamst@man.dtu.dk and thomassejr@gmail.com 
planning problem $[1,4,5]$. (2) Otherwise a Periodic VRPTW is solved, i.e., plans are made for several days, but regularity constraints are ignored $[2,6,7]$.

We propose a branch-and-price (BP) algorithm for the full long-term home care scheduling problem. The pricing problem generates a one-day plan for an employee, and the master problem merges the plans with respect to regularity constraints. The method is capable of generating plans with up to 44 visits during one week. This truly illustrates the complexity of the problem.

\section{Exact solution algorithm}

The problem considers a given period of time consisting of $L \in \mathbb{N}$ days. Time is discretized into time steps, which together span $L$. The set of employees is denoted $E$. For each employee $j \in E$ is given a set of days $H_{j}$ and time windows $\left[a_{j h}, b_{j h}\right], h \in$ $H_{j}$, that specify the work hours of $j$.

The set of visits is denoted $V$, the set of citizens is $C$, and the set of visits at citizen $c$ is $V_{c} \subseteq V$. Each visit $j \in V$ is repeated after $p_{j}$ amount of time. The visit repetitions are scheduled independently of each other and are denoted activities. Let $A$ be the set of all activities and $A_{j} \subseteq A$ the set of activities for visit $j \in V$. Two consecutive activities from visit $j$ are denoted $(i, k) \in A_{j}$. An activity $i \in A$ has attached a prioritized list of employees to conduct the activity, denoted $p r_{i}$. The prioritized list represents the skill set requirement. The duration of $i \in A$ is $d_{i}$ and the time window is $\left[a_{i}, b_{i}\right]$. The travel time between two activities $i, j \in A$ is $c_{i j} \geq 0$. Finally, busyness, i.e., the amount of time employee $j$ is late for conducting visit $i$, is denoted $b_{i j} \geq 0$.

Dantzig-Wolfe decomposing the problem results in a pricing problem, which generates a daily schedule for a given employee on a given day, and a master problem, which merges the daily schedules into an overall feasible solution.

The daily schedule for an employee is denoted a path and contains an ordered list of activities with attached starting times. The overall solution consists of paths for appropriate employees on appropriate days, covering all activities. Let $s_{i} \in \mathbb{N}$ denote the starting time of activity $i \in A$. Recall that the objective function consists of travel time, quality of service, and busyness. We aggregate these into a single, weighted objective function. Let $\mathbf{w}$ be the non-negative weight vector. The objective function which is to be minimized consists of:

1. Travel time (TT) between activities.

2. Busyness (B), i.e., how late an employee $j$ is for conducting an activity $i$ with respect to travel times and time windows.

3. Employee priority (EP).

4. Employee regularity (ER), i.e., the number of different employees at a citizen.

5. Visit regularity (VR), i.e., if the time between two consecutive activities $(i, k) \in$ $A_{j}$ differs from $p_{j}$. 
Let $p$ be a path and $P$ the set of all generated paths. Let $x_{p} \in\{0,1\}$ denote whether or not path $p$ is part of the solution, and let $u_{i k} \geq 0$ denote the difference in the starting times between two consecutive activities $(i, k) \in A_{j}$. Each path $p$ has a number of constants attached: $\delta_{B p}^{i j} \geq 0$ denotes the amount of busyness for employee $j$ and activity $i, \delta_{p}^{i} \in\{0,1\}$ denotes if activity $i$ is visited, $\delta_{s p}^{i} \geq 0$ denotes the start time at activity $i, \delta_{p}^{i j} \in\{0,1\}$ denotes if employee $j$ visits activity $i$ in the path, and $\delta_{p}^{j h} \in\{0,1\}$ denotes if the path is generated for employee $j$ on day $h \in H_{j}$. The master problem is formulated as:

$$
\begin{array}{cl}
\min \sum_{p \in P} \sum_{i \in A}\left(\sum_{k \in A} w^{T T} c_{i k} \delta_{p}^{i} \delta_{p}^{k} x_{p}+\sum_{j \in E}\left(w^{B} \delta_{B p}^{i j} x_{p}+w^{E P} p r_{i}(j) \delta_{p}^{i j} x_{p}\right)\right)+ \\
\sum_{c \in C} \sum_{j \in E} w^{E R} y_{c}^{j}+\sum_{j \in V} \sum_{(i, k) \in A_{j}} w^{V R} u_{i k} & \\
\text { s. t. } & \forall i \in A \\
\sum_{p \in P} \delta_{s p}^{i} x_{p}=1 & \forall j \in V, \forall(i, k) \in A_{j} \\
\sum_{p \in P} \delta_{s p}^{k} x_{p}-\left(\sum_{p \in P} \delta_{s p}^{i} x_{p}+p_{j}\right) \leq u_{i k} \delta_{s p}^{k} x_{p} \leq u_{i k} & \forall j \in V, \forall(i, k) \in A_{j} \\
\sum_{p \in P} \delta_{p}^{i j} x_{p} \leq y_{c}^{j} & \forall c \in C, \forall v \in V_{c}, \\
\sum_{p \in P} \delta_{p}^{j h} x_{p} \leq 1 & \forall i \in A_{v}, \forall j \in E \\
x_{p} \in\{0,1\} & \forall j \in E, \forall h \in H_{j} \\
u_{i k} \geq 0 & \forall p \in P \\
y_{c}^{j} \in\{0,1\} & \forall j \in V, \forall(i, k) \in A_{j}
\end{array}
$$

The objective function (1) minimizes a weighted sum of travel times, busyness, employee priorities, employee regularity and visit regularity. Constraints (2) ensure that every activity is visited. Constraints (3) and (4) measure visit regularity. Constraints (5) measure employee regularity. Constraints (6) ensure that at most one path per employee per day is part of a solution. The number of columns in the master problem is reduced by fixing daily visits to days: if a visit must be repeated every day, then the corresponding activities are fixed to day 1, 2, 3, etc., respectively. The pricing problem only allows such activities to be part of paths on appropriate days.

Let $\pi_{i}^{(2)} \in \mathbb{R}$ be the dual of constraints $(2), \pi_{i k}^{(3)} \leq 0$ the dual of $(3), \pi_{i k}^{(4)} \leq 0$ the dual of (4), $\pi_{i c j}^{(5)} \leq 0$ the dual of (5), and $\pi_{j h}^{(6)} \leq 0$ the dual of (6). The pricing problem is solved for each employee $j \in E$ on each day $h \in H_{j}$. The reduced cost of visiting activity $i \in A_{v}, v \in V_{c}, c \in C$ is:

$$
\bar{c}_{j h}^{i}=w^{E P} p r_{i}(j)-\pi_{i}^{(2)}-\pi_{i c j}^{(5)}- \begin{cases}s_{i}\left(\pi_{i k}^{(3)}-\pi_{i k}^{(4)}\right) & \exists k \in A:(i, k) \in A_{v} \\ 0 & \text { otherwise }\end{cases}
$$


Activity $i \in A$ is visited exactly once, hence the reduced cost for employee $j \in E$ on day $h \in H_{j}$ is defined as:

$$
\bar{c}_{j h}=\sum_{i \in A}\left(\bar{c}_{j h}^{i}+w^{B} b_{i j}+\sum_{k \in A} w^{T T} c_{i k}\right) \leq \pi_{j h}^{(6)}
$$

Recall that $b_{i j} \geq 0$ is the amount of busyness for employee $j$ at activity $i$ and that $c_{i k} \geq 0$ is the travel time between activities $i, k \in A$. Now, $\pi_{j h}^{(6)}$ is a constant, so if the pricing problem generates a path where $\bar{c}_{j h} \leq \pi_{j h}^{(6)}$, then the path has negative reduced cost and the corresponding column is added to the master problem. The pricing problem is recognized as a shortest path problem with time constraints and potentially negative edge weights. This is also denoted the Elementary Shortest Path Problem with Resource Constrained (ESPPRC), which is NP-hard. We solve the problem to optimality using the labeling algorithm in [3]. Initially, we try to solve the pricing problem heuristically using the labeling algorithm, where only a single label is stored at each activity.

Branching is necessary when the optimal solution in a branch node is fractional. The following strategy is finite and eventually ensures a feasible solution. Fractional solutions occur when:

An employee $j$ visits a citizen $c$ fractionally. Two branching children are generated with added cut: $\left(y_{c}^{j}=0\right)$ resp. $\left(y_{c}^{j}=1\right)$.

An activity $i$ is visited by several employees $j, j^{\prime}$ or on several days $h, h^{\prime}$. Two branching children are generated with rules: $\left(\sum_{p \in P} \delta_{p}^{i} \delta_{p}^{j h} x_{p}=0\right)$ resp. $\left(\sum_{p \in P} \delta_{p}^{i} \delta_{p}^{j^{\prime} h^{\prime}}\right.$ $\left.x_{p}=0\right)$. The pricing problem ensures that employee $j$ (resp. $j^{\prime}$ ) never visits activity $i$ on day $h$ (resp. $h^{\prime}$ ).

An employee $j$ travels on edges $(i k),\left(i k^{\prime}\right)$ on a given day $h$ a fractional number of times. Let $i$ be the first activity, from which employee $j$ travels on different edges or at different times. Let constant $\delta_{p}^{s_{i k}}$ denote whether or not path $p$ travels from $i$ to $k$ at time $s_{i k}$. Two branching children are generated with the following rules: $\left(\sum_{p \in P} \delta_{p}^{j h} \delta_{p}^{s_{i k}} x_{p}=0\right)$ resp. $\left(\sum_{p \in P} \delta_{p}^{j h} \delta_{p}^{s_{i k^{\prime}}} x_{p}=0\right)$. The pricing problem ensures that employee $j$ never travels from $i$ to $k$ (resp. $k^{\prime}$ ) at time $s_{i k}$ (resp. $s_{i k^{\prime}}$ ) on day $h$.

The branching rules do not complicate the pricing problem, because they either consider different columns $\left(y_{c}^{j}\right)$ or consist of rules, which can trivially be handled by forbidding appropriate extensions in the labeling algorithm.

\section{Computational Results}

The BP algorithm is implemented using the framework COIN Bcp [8] and tested on an Intel $2.13 \mathrm{GHz}$ Xeon CPU with 4 cores and 8 GB RAM. Note that test results stem from using a single core. CPLEX 12.1 is used as standard MIP solver. 
The BP algorithm is tested on a number of real-life benchmark instances provided by Papirgrden, a home care center in Funen, Denmark. Time is discretized into either 5 or 10 minute time steps. The objective weights are as follows: $W^{T T}=$ $500, w^{B}=750, w^{E P}=0, w^{E R}=750 \cdot 5 / \tau$ and $w^{V R}=50$, where $\tau$ denotes the size of a time step. We have tested two algorithms: (1) The exact BP algorithm as described and (2) a heuristic BP algorithm where columns are only generated heuristically.

\begin{tabular}{|c|c|c|c|c|c|c|c|c|c|c|c|}
\hline Instance & Maste & Toutent & Tree & Gap & Sol. & Time & Maste & oblem & Tree & Sol. & Tim \\
\hline & Cols & Rows & Size & & Value & Sec. & Cols & Rows & Size & Value & $\mathrm{Sec}$ \\
\hline $1-20-5$ & 6327 & 199 & 1 & 0.00 & 27000.0 & 3.97 & 1794 & 199 & 1 & 38750.0 & 0.75 \\
\hline $1-25-5$ & 8671 & 250 & 4687 & 0.79 & 29750.0 & 1801.33* & 608 & 250 & 1 & 44500.0 & 0.18 \\
\hline $1-30-5$ & 12883 & 295 & 2079 & 3.17 & 38000.0 & $1801.97 *$ & 2852 & 295 & 1 & 86000.0 & 1.45 \\
\hline $1-40-5$ & 28365 & 397 & 171 & 0.00 & 43000.0 & 812.94 & 903 & 397 & 1 & 67500.0 & 0.55 \\
\hline $1-50-5$ & 31480 & 493 & 27 & 110.91 & 87000.0 & $1803.35^{*}$ & 1235 & 493 & 1 & 97500.0 & 1.45 \\
\hline $1-80-5$ & 8737 & 787 & 1 & 333.89 & 259250.0 & $1906.50 *$ & 2954 & 787 & & 259250.0 & 10.96 \\
\hline $2-20-5$ & 8917 & 346 & 5 & 0.00 & 27000.0 & 8.58 & 1917 & 346 & 1 & 31750.0 & 0.66 \\
\hline $2-25-5$ & 10089 & 432 & 2405 & 0.94 & 29750.0 & $1803.39 *$ & 1416 & 432 & 3 & 34750.0 & 0.27 \\
\hline $30-5$ & 16383 & 512 & 695 & 1.41 & 38400.0 & $1803.29 *$ & 5637 & 512 & 481 & 73500.0 & 117.37 \\
\hline $2-80-5$ & 8261 & 1354 & 1 & 297.49 & 237500.0 & $1908.73 *$ & 12677 & 1354 & 185 & 237500.0 & 388.09 \\
\hline $1-20-10$ & 4877 & 199 & 59 & 0.00 & 17250.0 & 6.37 & 1286 & 199 & 1 & 24750.0 & 0.29 \\
\hline $1-25-10$ & 5507 & 250 & 277 & 0.00 & 18125.0 & 27.36 & 324 & 250 & 1 & 29625.0 & 0.11 \\
\hline $1-30-10$ & 10934 & 295 & 2449 & 0.32 & 25300.0 & $1801.07 *$ & 1845 & 295 & 1 & 54250.0 & 0.71 \\
\hline $1-40-10$ & 14037 & 397 & 63 & 0.00 & 27750.0 & 90.16 & 733 & 397 & 1 & 48750.0 & 0.32 \\
\hline $1-50-10$ & 19907 & 493 & 3 & 0.00 & 36500.0 & 337.04 & 1002 & 493 & 1 & 67500.0 & 0.71 \\
\hline $5-10$ & 19912 & 544 & 3 & 0.00 & 60375.0 & 123.64 & 1409 & 544 & 1 & 86875.0 & 0.69 \\
\hline $1-58-10$ & 40434 & 571 & 111 & 73.60 & 64450.0 & $1803.82 *$ & 2270 & 571 & 1 & 106650.0 & 1.89 \\
\hline $1-80-10$ & 19711 & 787 & 1 & 224.63 & 161100.0 & $1847.78 *$ & 2255 & 787 & 1 & 161100.0 & 4.44 \\
\hline $2-20-10$ & 5913 & 346 & 39 & 0.00 & 17250.0 & 9.19 & 1770 & 346 & 1 & 20750.0 & 0.43 \\
\hline $2-25-10$ & 6594 & 432 & 81 & 0.00 & 18125.0 & 16.30 & 714 & 432 & 3 & 23125.0 & 0.15 \\
\hline $2-30-10$ & 10109 & 512 & 1245 & 0.20 & 25200.0 & $1802.52 *$ & 4585 & 512 & 751 & 48000.0 & 115.49 \\
\hline $2-40-10$ & 15937 & 684 & 77 & 0.00 & 27250.0 & 162.93 & 3267 & 684 & 7 & 37500.0 & 1.00 \\
\hline $2-50-10$ & 25457 & 850 & 21 & 0.00 & 35750.0 & 654.53 & 6914 & 850 & 191 & 61250.0 & 58.38 \\
\hline $2-55-10$ & 29331 & 936 & 49 & 133.70 & 80625.0 & $1841.08 *$ & 6090 & 936 & 67 & 80625.0 & 21.94 \\
\hline $2-58-10$ & 21593 & 984 & 31 & 160.61 & 96750.0 & $1826.54 *$ & 7242 & 984 & 143 & 96750.0 & 76.81 \\
\hline 2-80-10 & 22136 & 1354 & 3 & 201.76 & 149750.0 & $2475.74 *$ & 8552 & 1354 & 221 & 149500.0 & 265.50 \\
\hline
\end{tabular}

Table 1 Results for the exact BP (left hand side) and heuristic BP (right hand side) for instances named " $|E|-|A|-\tau$ ", $\tau=$ time step size. Tests marked with * exceeded the 30 minute time bound.

Test results are summarized in Table 1. As can be seen from the left hand size of the Table, only few instances with 5 minute time steps can be solved to optimality within half an hour. A coarser discretization helps, but the BP algorithm still suffers from a large time usage. The number of columns is large, which is caused partly by large time windows and partly by busyness, i. e., that time windows may be violated. The tree size grows very large for some instances not solved to optimality, hence branching also constitutes a bottleneck. As can be seen from the right hand side of the Table, the heuristic BP algorithm is generally faster. Few instances suffer from large tree sizes and many columns, but the far majority of instances are solved 
in seconds. Unfortunately, the objective values generally suffer from the heuristic approach.

Improving the exact BP approach would require methods for reducing the number of columns and for improving the bounds to prune larger parts of the tree. The authors attempted stabilizing the value of dual variables using an interior point method [9], but with no avail. Other stabilization methods could be investigated, as better values for the dual variables could reduce the number of generated columns. The authors also tried different primal and incumbent heuristics for improving the bounds with little luck. Future work could continue on such heuristics or on changing the branching strategy.

\section{Conclusion}

In this paper, we presented a BP algorithm for the long-term home care scheduling problem. The $N P$-hard pricing problem consisted of calculating a work plan on a given day for a given employee and the master problem merged the plans into an overall optimal solution. The BP algorithm was tested on a number of real-life instances and was capable of only solving smaller instances due to the large number of combinations of visits, visit times and employees. This truly illustrates the complexity of the problem.

Acknowledgements We would like to thank Papirgden for sharing knowledge and real-life data for home care scheduling, and Villum-Kann-Rasmussen foundation for supporting this work.

\section{References}

1. Begur S. V., Miller D. M., Weaver J. R.: An Integrated Spatial DSS for Scheduling and Routing Home-Health-Care Nurses. Interfaces 27, 35-48 (1997)

2. Claassen G.D.H., Hendriks Th.H.B.: An application of Special Ordered Sets to a periodic milk collection problem. European Journal of Operational Research 180, 754 - 769, (2007)

3. Dumitrescu I., Boland N.: Improved preprocessing, labelling and scaling algorithms for the weight-constrained shortest path problem. Networks 42, 135-153 (2004)

4. Eveborn P., Flisberg P., Rönnqvist M.: Laps Care - an operational system for staff planning of home care. European Journal of Operational Research 171, 962-976 (2006)

5. Hansen A. D., Rasmussen M. S., Justensen T., Larsen J.: The Home Care Crew Scheduling Problem. In 1st International Conference on Applied Operational Research volume 1 of Lecture Notes in Management Science, 1-9 (2008)

6. Hemmelmayr V., Doerner K., Hartl R., Savelsbergh M.: Delivery strategies for blood products supplies. OR Spectrum 31, 707-725 (2008)

7. Jang W., Lim H. H., Crowe T. J., Raskin G., Perkins T. E.: The Missouri Lottery Optimizes Its Scheduling and Routing to Improve Efficiency and Balance. Interfaces 36, 302-313 (2006)

8. Lougee-Heimer R.: The Common Optimization INterface for Operations Research. IBM Journal of Research and Development 47, 57-66 (2003)

9. Rousseau L.-M., Gendreau M., Feillet D.: Interior point stabilization for column generation. Operations Research Letters 35, 660-668 (2007) 